\title{
Bicuspid Aortic Valve: Progression of Stenosis and Clinical Relevance
}

\author{
Saqib Masroor ${ }^{1}$ \\ ${ }^{1}$ University of Toledo Medical Center
}

September 25, 2021

Title: Bicuspid Aortic Valve: Progression of Stenosis and Clinical Relevance

Running Head: Progression of Stenosis in Bicuspid Aortic Valve

Authors: Saqib Masroor, MD, MBA, MHS

University of Toledo College of Medicine and Life Sciences, Department of Surgery, Division of Cardiothoracic Surgery

Meeting Presentation: None

Disclosure: None

Word Count: 717

\section{Corresponding Author:}

Saqib Masroor, MD, MHS

Chief, Division of Cardiothoracic Surgery

University of Toledo College of Medicine and Life Sciences

3000 Arlington Avenue, Toledo, Ohio 43614

Phone: 419-383-5150

Fax: 419-383-3149

Bicuspid aortic valve (BAV) is the most common congenital anomaly and nearly $50 \%$ of people with BAV require aortic valve replacement (AVR) about 10-20 years earlier than those with tricuspid aortic valves (TAV)1. It is however not clearly known if aortic stenosis (AS) progresses at a faster pace in BAV vs TAV. Studies so far reported in the literature have been inconclusive and marked by relatively small number of patients and short to mid-term follow-up (2-6 years $)^{1-2}$.

In this issue of the Journal of Cardiac Surgery, Shang et al ${ }^{3}$ report on 59 patients with BAV, matched 1:3 with TAV patients with mild or moderate aortic stenosis, and conclude that the progression to severe AS in both groups is similar. However, BAV was associated with an increased risk of AVR. This is an elegant study, however there are some issues with its methodology and design of the study. The authors write in the introduction that "... older age, male sex, coronary artery disease, plasma level of oxidized phospholipids,... have been associated with more rapid prognosis". Yet, the study did not investigate the incidence of coronary artery disease, dyslipidemia or metabolic syndrome in the two groups. Moreover, the BAV group is 20 years younger than the TAV group. Hence the influence of these unmatched variables on the outcomes cannot be ruled out. 
Another possible source of error is inconsistent echocardiographic assessment of AS. Earliest echocardiograms are from 2005 and the latest one is from 2020. Echocardiography was not done for the purposes of the study and hence there may not have been a consistency in the assessment of the AS severity. One of the three hemodynamic parameters necessary for AS severity assessment is peak jet velocity. Accurate measurement of peak jet velocity requires using multiple acoustic windows to determine the highest velocity and it requires particular attention to patient positioning and adjustment of ultrasound transducer position and angle. Misalignment of ultrasound beam and AS jet results in underestimation of jet velocity and even greater underestimation of the pressure difference. ${ }^{4}$ The reason I raise this issue is the fact that while patients with trace or no AS were excluded from the study, $12 \%$ of BAV and $5 \%$ of TAV patients ended up with no AS at the study end.

A more consistent and accurate way of following progression of AS would be desirable.

And one candidate for this is contrast-enhanced CT imaging to assess aortic valve calcification, which has been shown to have strong correlation with AS. ${ }^{5}$ However others have shown that this correlation is strong in TAV patients of all ages, but in BAV patients, it only holds strong in patients older than 51 years of age. Younger patients with BAV can have significant AS without an associated increase in AV calcification. ${ }^{6}$

Finally, no account of BAV is complete without a mention of associated aortopathy. Shang does not include data on the size of the ascending aorta in the patients. In fact, despite similar progression AS, BAV patients exhibit a higher incidence of surgery. This could very well be due to the progressive enlargement of aorta requiring aortic surgery and concomitant AVR even with moderate AS. Indeed Song et $\mathrm{al}^{7}$ have shown that in BAV patients followed up for 6 years, while 12\% patients underwent AVR, another 10\% patients underwent replacement of aorta.

Overall, Shang et al should be complimented on putting together a valuable series of patients with BAV and highlighting their progression of AS. It is becoming increasingly clear that the rate of progression of AS is the same in BAV and TAV patients, but they still require more surgical intervention, whether it be AVR or aortic replacement or a combination thereof. While academically we may continue to question whether to perform closer surveillance of BAV patients (with mild AS) than TAV or not, the more important point for us clinicians is to look at BAV and aorta as one entity. It is preferable that surgeons follow these asymptomatic BAV patients, as they are better trained and equipped to treat both the valvular disease and aortopathy. These patients do require more frequent and earlier interventions. And because failure of timely intervention in these patients can be catastrophic. While BAV occurs in 1\% of the population, it comprises 10-15\% of patients with aortic dissection! ${ }^{8}$

\section{REFERENCES}

1. Shen M, Tastet L, Capoulade R, et al. Effect of bicuspid aortic valve phenotype on progression of aortic stenosis. European Heart J - Cardiovasc Imaging 2020:21;727-34

2. Ryu DR, Park SJ, Han H, et al. Progression rate of aortic valve stenosis in Korean patients. J Cardiovasc Ultrasound 2010:18:127-33

3. Shang M, Kahler-Quesada A, Mori M, et al. Progression of aortic stenosis in patients with bicuspid aortic valve.

4. Baumgartner H, Hung J, Bermego J, et al. Recommendations on the echocardiographic assessment of aortic valve stenosis: A focused update from the European Association of Cardiovascular imaging and the American Society of Echocadiograpny. J Am Soc Echocardiogr 2017;30:372-92

5. Pawade T, Sheth T, Guzzetti E, Dweck MR and Clavel M-A. Why and how to measure aortic valve calcification in patients with aortic stenosis. J Am Coll Cardiol Img 2019;12:1835-48

6. Shen M, Tastet L, Capoulade R, et al. Effect of age and aortic valve anatomy on calcification and haemodynamic severity of aortic stenosis. Heart 2017;103(1):32-9 
7. Song S, Seo J, Cho I, et al. Progression and outcomes of non-dysfunctional bicuspid aortic valve: Longitudinal data from a large Korean bicuspid aortic valve registry. Front Cardiovasc Med 2020;7:603323 doi: $10.3389 /$ fcvm. 2020.603323

8. Edwards WD, Leaf DS and Edwards JE. Dissecting aortic aneurysm associated with congenital bicuspid aortic valve. Circulation 1978;57(5):1022-5 